\title{
Thoracomyoplasty for Chronic Empyema and Osteoradionecrosis of the Chest Wall
}

\author{
Jeong In Hong, M.D., Hong Ju Shin, M.D., Ph.D., Won-Min Jo, M.D., Ph.D., Jae Seung Shin, M.D., Ph.D., \\ Jinwook Hwang, M.D., Ph.D.
}

Department of Thoracic and Cardiovascular Surgery, Korea University Ansan Hospital, Korea University College of Medicine, Ansan, Korea

\section{ARTICLE INFO}

Received June 26, 2020

Revised August 25, 2020

Accepted September 3, 2020

Corresponding author

Jinwook Hwang

Tel $82-31-412-4290$

Fax 82-31-414-3249

E-mail znuke75@korea.ac.kr

ORCID

https://orcid.org/0000-0003-4940-165X

\begin{abstract}
Herein, we report a case in which thoracomyoplasty was performed to manage chronic postlobectomy empyema (PLE). A 54-year-old male patient with a surgical history of right upper lobectomy and thymectomy 35 years previously who had undergone adjuvant radiotherapy presented with purulent discharge on the anterior chest wall. The patient was diagnosed with chronic PLE with ascending infection and concurrent osteonecrosis of the parasternum. Proper drainage was performed for local infection control and the dead spaces were successfully closed with muscle flaps. There have been no complications to date.
\end{abstract}

Keywords: Pleural empyema, Osteoradionecrosis, Thoracoplasty, Myocutaneous flap

\section{Case report}

A 54-year-old male patient presented with febrile sensation and anterior chest wall pain with an erythematous ulcerative plaque in the right parasternal area that had become aggravated over a month despite antibiotic treatment prescribed at a local clinic. A swab culture was done, which led to the identification of Citrobacter koseri. The patient was admitted to the general ward for further workup and initiated on intravenous (IV) ciprofloxacin (400 mg every 12 hours).

The patient had undergone right upper lobectomy and thymectomy 35 years ago via posterolateral thoracotomy followed by adjuvant radiotherapy. A chest computed tomography scan revealed chronic empyema with a right apical calcified sac with an air-fluid level and chest wall abscess, for which continuity to the pleural space could not be excluded (Fig. 1).

An elliptical skin incision was made around the fistula orifice at the second and third intercostal spaces (ICSs) along the right side of the sternum at the costosternal junction. After removing the infected bony chest wall, we entered the empyema cavity and evacuated the pus and necrotic materials. A sump drainage tube was inserted into the cavity for subsequent irrigation and drainage.
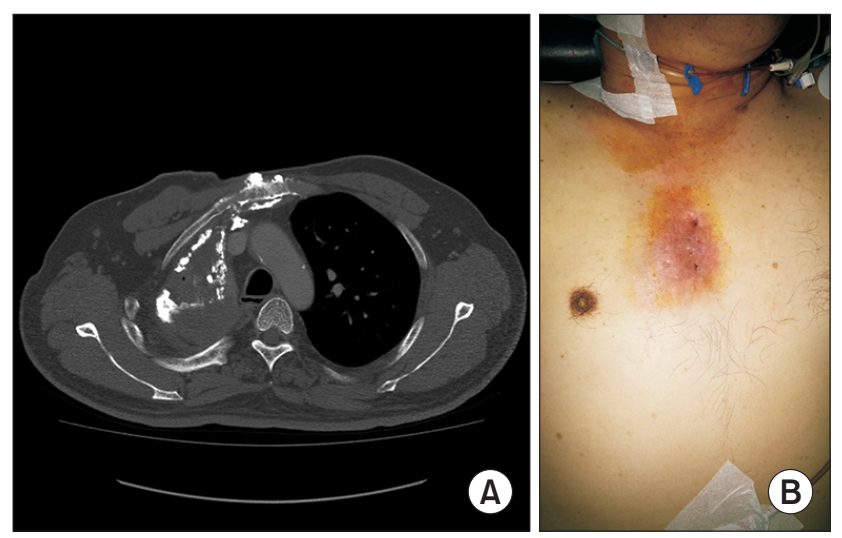

Fig. 1. (A) Preoperative computed tomography showing chronic empyema with a right apical calcified sac with an air-fluid level. (B) An ulcerative skin lesion in the anterior chest wall that was suspicious for a fistula.

Treatment with IV antibiotics was continued, and daily chest tube irrigation with $1 \mathrm{~L}$ of taurolidine-mixed purified water was performed for local infection control. Subsequently, a pleural fluid culture study was performed every 5 days, and no bacterial growth was observed. However, the patient remained febrile for 36 days after the initial operation; thus, we delayed the second stage of the operation. After discontinuing the irrigation using the chemical 

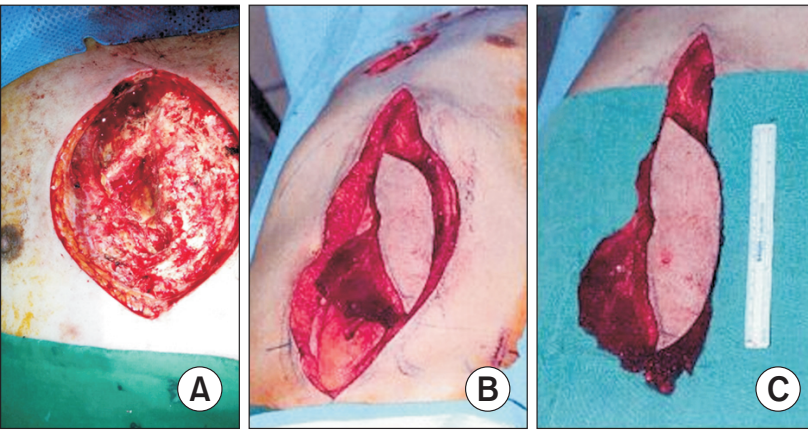

Fig. 2. (A) Anterior chest wall defect after removing the infected soft tissue and costosternal junction. (B, C) Vertical rectus abdominis myocutaneous flap harvesting and transposition.

agent, the patient was afebrile and in a suitable condition for thoracomyoplasty. The first stage of the operation included full thoracotomy and resection of the second to fifth ribs. The thoracic cavity was entered and inspected through the fourth ICS periosteal bed. Severe visceral pleural adhesion to the chest wall was seen inferior to the fifth ICS level, and the cavitary empyema wall superior to fourth ICS level was dissected using a rongeur and Mayo scissors to confirm the previously inserted intracavitary sump drain. A serratus anterior (SA) muscle flap was harvested and transposed inside the chest through the ICS and sutured on the anterior and posterior chest wall to obliterate the apical dead space.

The second stage of the operation was performed 14 days later for definitive closure of the surgical wound. The interval between the stages of surgery provided adequate time to assess the viability of the SA flap and the sufficiency of space obliteration, as well as to confirm a negative microbiology report to ensure that there would be no local recurrence of infection. Here, a pedicled vertical rectus abdominis myocutaneous (VRAM) flap was utilized to fill in the right anterior chest wall soft tissue and skin defect from the first exploratory thoracotomy operation. The SA flap transposed in the previous operation was identified in the field, which appeared fresh with a healthy blood supply. The VRAM flap preparation and coverage were carried out by a plastic surgeon. The flap was passed through the subcutaneous tunnel to reach the space where the pericardium was exposed (Fig. 2).

There were no postoperative complications. The Jackson-Pratt drain was removed on postoperative day 12 , and the patient was discharged home on postoperative day 21 . He was then followed up at the outpatient clinic until 17 months postoperatively; there was no recurrence of infection, and no impairment of activities of daily living (Fig. 3).

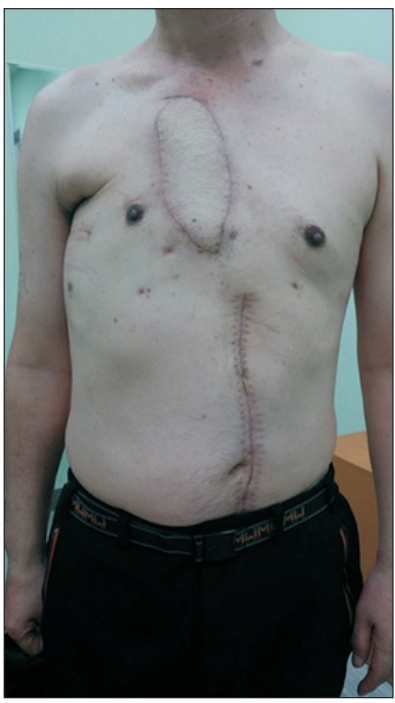

Fig. 3. Postoperative follow-up at the outpatient clinic.

\section{Discussion}

The study was approved by the Institutional Review Board (IRB approval no., 2020AS0278) and the requirement for informed consent was waived. Post-pulmonary resection empyema is a challenging condition to manage. The incidence of a residual space after lobectomy at discharge ranges from $5 \%$ to $21 \%$, and infection develops in approximately $2 \%$ of these patients [1]. The incidence of postpneumonectomy empyema (PPE) varies from $2.2 \%$ to $16 \%$, and the mortality rate of PPE is high if it is associated with a fistula [2]. The primary principles of PPE and postlobectomy empyema management are drainage of the abscess, local and systemic infection control, and complete thoracic space obliteration to prevent recurrent infection. The modern management of empyema started during World War I, when the Empyema Commission created by the US Army realized the importance of the negative pleural pressure and closed drainage [3]. Open pleural drainage, such as the Eloesser flap or Clagett procedure, is a classic approach. Recently accumulated evidence suggests that the intrathoracic application of vacuum-assisted closure is a possible adjunct to achieve successful chest closure [4]. Various thoracoplasty techniques have been described as procedures to obliterate the thoracic cavity, such as intrapleural thoracoplasty by Schede (1890), extrapleural thoracoplasty popularized by Alexander (1937), and plombage thoracoplasty for better cosmetic results by Andrews (1949). These techniques are still used currently with or without modifications [5]. Other options, such as filling 
the cavity with an antibiotic solution with watertight closure, and muscular or omental flap transposition, have also been introduced [2]. Owing to the small number of patients and heterogeneity of the locoregional anatomy, there have been no prospective randomized studies, and most of the current surgical approaches are mainly based on experts' opinions $[3,6]$.

Chest wall osteoradionecrosis (ORN) can occur after high-dose radiotherapy in patients with lung and breast cancers. Although its incidence has been decreasing due to improvements in radiation therapy devices and betterplanned treatment regimens, ORN is a severe complication that requires urgent attention. The damage is primarily mediated by reactive oxygen species that alter the soft tissue and microvasculature environment, leading to tissue fibrosis and necrosis. The slow pathologic progression often goes unnoticed until the development of full-thickness necrosis and skin ulceration with superinfection. Moreover, soft tissue edema, rib fractures, brachial plexopathy, cardiac morbidity/mortality, secondary cancer such as sarcoma, and pneumonitis can develop. When left untreated, the loss of necrotic soft tissue and ribs will expose the thoracic viscera, resulting in empyema. Surgical resection is the treatment of choice, and reconstruction is indicated once the local infection is eradicated. Large defects $(>3-4$ ribs) of the anterior chest wall may increase the risk of substantial chest flail in patients; hence, mechanical prosthesis implementation to ensure stability is required. A contrasting opinion exists that the radiation-injured mediastinum would have been fixed in place without the risk of paradoxical movement; therefore, no prosthesis is necessary, as it can be a source of infection $[7,8]$.

In our case, the chronic empyema 35 years after lobectomy and thymectomy was associated with anterior chest wall ORN and pleurocutaneous fistula. Initial surgical debridement by anterior thoracotomy included resection of the pleura along with the infected chest wall, rib cartilage, internal thoracic artery, and vein. The sump drainage tube was positioned inside the empyema cavity, and daily irrigation through the tube was continued for 36 days to achieve a clean environment for thoracomyoplasty. A 2-staged surgical intervention was performed; the first operation was performed to obliterate the thoracic cavity with thoracic muscle flaps, and the second one was to cover the defect left from the initial anterior chest wall resection with a myocutaneous flap. Thoracoplasty for thoracic cavity reduction was performed by extrapleural resection of the second to fifth ribs, while the first rib resection was withheld to prevent shoulder girdle dysfunction. An SA muscle flap was used in this case due to its proximity to the apical dead space.

Thoracomyoplasty is a radical, mutilating operation that may result in undesirable cosmetic sequelae and impaired activities of daily life. Because relatively few patients need such procedures and considerable technical improvements have been made in minimally invasive approaches, thoracomyoplasty is seldom done these days. However, this procedure is still indicated in patients with a chronic pleural space infection where decortication alone is ineffective in inducing full-lung re-expansion. The overall mortality and success rates of thoracomyoplasty are believed to be approximately $5 \%$ and over $90 \%$, respectively, according to recent studies [3].

In the absence of standardized protocols for managing patients with chronic postlobectomy empyema with or without fistula, this reported case may remind surgeons of these classic procedures, along with the use of a myocutaneous flap and more contemporary techniques such as the vacuum-assisted closure application. A tailored approach for each individual may lead to a more favorable prognosis.

\section{Conflict of interest}

No potential conflict of interest relevant to this article was reported.

\section{ORCID}

Jeong In Hong: https://orcid.org/0000-0002-9660-7063

Hong Ju Shin: https://orcid.org/0000-0002-0731-3523

Won-Min Jo: https://orcid.org/0000-0002-0082-0098

Jae Seung Shin: https://orcid.org/0000-0001-8147-6665

Jinwook Hwang: https://orcid.org/0000-0003-4940-165X

\section{References}

1. Fournier I, Krueger T, Wang Y, Meyer A, Ris HB, Gonzalez M. Tailored thoracomyoplasty as a valid treatment option for chronic postlobectomy empyema. Ann Thorac Surg 2012;94:387-93.

2. Hysi I, Rousse N, Claret A, et al. Open window thoracostomy and thoracoplasty to manage 90 postpneumonectomy empyemas. Ann Thorac Surg 2011;92:1833-9.

3. Botianu PV, Botianu AM. Thoracomyoplasty in the treatment of empyema: current indications, basic principles, and results. Pulm Med 2012;2012:418514.

4. Haghshenasskashani A, Rahnavardi M, Yan TD, McCaughan BC. Intrathoracic application of a vacuum-assisted closure device in managing pleural space infection after lung resection: is it an option? In- 
teract Cardiovasc Thorac Surg 2011;13:168-74.

5. Stefani A, Jouni R, Alifano M, et al. Thoracoplasty in the current practice of thoracic surgery: a single-institution 10-year experience. Ann Thorac Surg 2011;91:263-8.

6. Krassas A, Grima R, Bagan P, et al. Current indications and results for thoracoplasty and intrathoracic muscle transposition. Eur J Cardiothorac Surg 2010;37:1215-20.
7. Makboul M, Salama Ayyad MA. Is myocutaneous flap alone sufficient for reconstruction of chest wall osteoradionecrosis? Interact Cardiovasc Thorac Surg 2012;15:447-51.

8. Raz DJ, Clancy SL, Erhunmwunsee LJ. Surgical management of the radiated chest wall and its complications. Thorac Surg Clin 2017;27: 171-9. 